\title{
Post Mortem Study of Alcoholic and Non-Alcoholic Liver Diseases in Ajmer Region
}

\author{
Vandana Porwal*, Neha Mishra, Deepali Jain and Seema Gupta \\ Department of Pathology, Jawahar Lal Nehru Medical College, Ajmer (Rajasthan) INDIA
}

\begin{abstract}
Background: Most of the chronic liver diseases even in advanced stage may cause no prominent clinical signs or symptoms. They either go undiagnosed or are found incidentally during general health check-ups, investigations for other diseases, surgery, or autopsy. The underlying causes of chronic liver diseases vary in different geographic areas and are based on various factors such as socioeconomic status, life style, diet, local or regional infections, and other endemic diseases. The aim of our study is to establish prevalence of silent liver diseases like NASH and alcoholic liver disease and its correlation with age, sex, lifestyle and other factors in local population.
\end{abstract}

Methods: 146 Post-mortem specimens of liver were received in Department of Pathology, J.L.N. Medical College, Ajmer from January 2018 to August 2018. 27 cases were excluded due to autolysis. Information regarding age, sex, marital status, place, food habit, alcoholic usage and previous history of any disease were collected in whenever cases possible. Processed tissue sections were stained and were analysed by descriptive statistics.

Result: Total 119 cases of liver were included. The age ranged from 11-90 years with mean age $42.14 \pm 13.12$ years. Majority of patients belonged to the 4th decade of life, followed by 5th decade with male preponderance (M:F ratio 5:1). Maximum number of cases was of steatosis with grade I and IV. Important findings included steatosis in 58 (48.74\%), steatohepatitis in 12 (10.08\%), chronic hepatitis in 36 $(30.25 \%)$, confluent necrosis in $8(6.72 \%)$, cirrhosis in $5(4.2 \%)$ cases.

Conclusion: Silent diseases of the liver like NASH are not uncommon. Steatosis and chronic hepatitis comprise a significant finding in our locality.

Keywords: Autopsy, Liver Disease, Steatosis, Chronic Hepatitis

\section{Introduction}

Liver diseases vary in different geographic areas and are based on various factors such as socio-economic status, life style, diet, local or regional infections and other endemic disease. ${ }^{[1]}$ Most of the chronic liver diseases even in advanced stages may cause no prominent clinical signs or symptoms and are undiagnosed or found incidentally during general checkups, investigations for other diseases or during autopsy. ${ }^{[2]}$ Alcohol abuse generally leads to three pathologically distinct liver diseases viz. fatty liver, hepatitis and alcoholic cirrhosis. ${ }^{[3]}$ One or all of the three can occur at the same time and in the same patient. ${ }^{[4]}$

According to the WHO, alcohol consumption accounts for $3.8 \%$ of the global mortality and $4.6 \%$ of DALYs. Liver disease represents 9.5\% of alcohol-related DALY's worldwide, while individual rates vary in different regions. Globally, the prevalence of NASH ranges from $6 \%$ to $35 \%$, with a median of $20 \%{ }^{[5]}$ Prevalence rates from China, India and Japan have been reported as 5\%, 5-28\% and 14\%, respectively. Prevalence in the US, using non-invasive tests is reported to be as high as $10-35 \%$ but only $3-5 \%$ using liver biopsy. ${ }^{[6]}$ Global prevalence of cirrhosis from autopsy studies ranges from $4.5 \%$ to $9.5 \%$ of the general population ${ }^{[7,8,9]}$. Hence, we estimate that more than fifty million people in the world, taking the adult population, would be affected with chronic liver disease. Globally, alcohol, NASH and viral hepatitis currently are the most common causative factors. Prevalence of cirrhosis is likely to be underestimated as almost a third of the patients remain asymptomatic. With the use of non-invasive tests like transient elastography, a more realistic picture could emerge in the near future. ${ }^{[10]}$

Hence the present study has been undertaken to establish prevalence of silent liver diseases like NASH and alcoholic liver disease and its correlation with age, sex, lifestyle and other factors in local population.

\section{Materials and Methods}

Total 146 Postmortem specimens of liver were received in Department of Pathology, J.L.N. Medical College, Ajmer from January 2018 to August 2018. 27 cases were excluded due to autolysis. The liver specimens were received as a part of examination of multiple viscera. Information regarding age, sex, marital status, place, alcoholic usage and previous history of any disease particularly liver disease 
etc., were collected from the first degree of the deceased during autopsy in which ever cases possible. All the bits were fixed in 10\% formalin. Processed tissue sections were stained with Haematoxylin and Eosin according to standard procedure. Special stains like Reticulin stain, Von Gieson stain (VG stain); Periodic Acid Schiff's (PAS) stain and Congo red stain were performed where necessary. The findings were recorded and the cases were analysed by descriptive statistics.

Inclusion criteria: All persons on whom medico legal autopsies had been done to ascertain the cause of death

Exclusion criteria: Autopsies on exhumed bodies.

\section{Result}

In the present study, total 119 cases of liver were included (Figure1). The age ranged from 11-90 years with mean age $42.14 \pm 13.12$ years. Majority of patients belonged to the 4 th decade of life, followed by 5 th decade. Male predominance was seen with M:F ratio of 5:1. Maximum number of cases was of steatosis with grade I and IV.

Steatosis - In our study steatosis was seen in 58 (48.74\%) cases with male to female ratio 3.8:1. Maximum number of cases seen in males in $4^{\text {th }}-5^{\text {th }}$ decade but in females seen in slightly earlier age group in $3^{\text {rd }}$ decade (Figure 2)

Steatosis was graded with maximum cases seen in grade I and IV (Table 1). ${ }^{[1]}$ Predominantly macro vesicular steatosis with fatty cyst formation seen.

Steatohepatitis - Steatohepatitis was seen in 12 cases (10.08\%). Maximum cases were seen in adult males between 31-40 years (Figure 2) and were characterised by steatosis with hepaticellular damage, inflammation and fibrosis. A scoring system for steatohepatitis was done with necroinflammatory grading of Grade 1 (mild), Grade 2 (moderate) to grade 3 (severe) (Table 2). ${ }^{[1]}$
Most patients with steatohepatitis were alcohol abusers, overweight or diabetic or have a combination of these attributes. The histological lesions of nonalcoholic steatohepatitis (NASH) were very severe compared with alcoholic steatohepatitis (ASH). The presence of abundant neutrophils and Mallory bodies should lead to a suspicion of alcohol abuse. Glycogen vacuolation of nucleus is common in non alcoholic steatohepatitis NASH. However, there was no significant difference in the histologic grade of fibrosis, inflammation or hepatocytic metamorphosis between NASH and alcoholic hepatitis. Stellate form fibrosis was characteristic of alcoholic hepatitis where as pericellular and perivenular types were common in NASH patients. Focal cell necrosis was rather intense and fatty deposits prominent in NASH patients. If alcoholic hepatitis is histologically suspected in non alcoholic patients, the possibility of NASH should always be considered.

Chronic hepatitis -A simple scoring system was done for chronic hepatitis (Table 3 ). ${ }^{[11]}$ We reported 36 cases of chronic hepaptitis. The common affected age was 31-40 years with preponderance of males $(88.63 \%)$ (Figure 2$)$. The mildest form of chronic hepatitis showed confinement of the inflammatory infiltrate in the portal tracts. Margins of portal tract were regular and clean. Cases of interface hepatitis were also seen, the inflammatory cells extended from the portal tracts into the adjacent parenchyma and there was destruction of hepatocytes. In our study majority of the cases had mild to moderate portal inflammation and mild or localized interface hepatitis. We also encountered 8 cases of confluent necrosis with maximum cases seen in 21-50 years age male (Figure 3 ).

Liver cirrhosis - 5 cases of cirrhosis were seen in elderly males only. Hepatocellular changes in the form of regenerative hyperplasia were noted. Micronodular cirrhosis was more common as compared to macronodular cirrhosis (Figure 4).

Table 1: Grading of Steatosis ${ }^{[11]}$

\begin{tabular}{|l|l|l|}
\hline Grade & No. of cases $(\mathbf{n})$ & $\%$ \\
\hline I & 17 & 29.31 \\
\hline II & 13 & 22.41 \\
\hline III & 13 & 22.41 \\
\hline IV & 15 & 25.86 \\
\hline Total & $\mathbf{5 8}$ & \\
\hline I & When micro and macrovesicularsteatosis affected 5-25\% of the liver parenchyma \\
\hline II & When 26-50\% of the parenchyma was affected \\
\hline III & When 51-75\% was affected \\
\hline IV & When more than 75\% of the parenchyma was involved \\
\hline
\end{tabular}


Table 2: A Scoring system for Steatohepatitis ${ }^{[11]}$

\begin{tabular}{|c|c|}
\hline Necroinflammatory & \\
\hline Grade I (mild) & $\begin{array}{l}\text { Steatosis ( mainly macrovesicular) involving up to } 66 \% \text { of lobules; occasional ballooned } \\
\text { perivenular hepatocytes; scattered neutrophils with or without lymphocytes; no or mild } \\
\text { chronic portal inflammation }\end{array}$ \\
\hline Grade 2 (moderate) & $\begin{array}{l}\text { steatosis of anydegree; obvious ballooning (mainly perivenular); intralobular neutrophils, } \\
\text { may be associated with perivenularpericellular fibrosis if evident; mild to moderate portal and } \\
\text { intralobular chronic inflammation }\end{array}$ \\
\hline Grade 3 (severe) & $\begin{array}{l}\text { Panlobularsteatosis; obvious perivenular ballooning and disarray; marked lobular } \\
\text { inflammation; neutrophils may be concentrated in perivenular areas of ballooning and in } \\
\text { areas of pericellular fibrosis if evident. Portal inflammation mild or moderate }\end{array}$ \\
\hline Fibrosis staging & \\
\hline Stage 1 & Pericellular fibrosis in perivenular areas, focal or extensive \\
\hline Stage 2 & As above, plus focal or extensive Periportal fibrosis \\
\hline Stage 3 & Bridging fibrosis, focal or extensive \\
\hline Stage 4 & Cirrhosis \\
\hline
\end{tabular}

Table 3: Staging and Grading of cases of Chronic Hepatitis ${ }^{[11]}$

\begin{tabular}{|l|l|l|}
\hline Total scores & Staging \& Grading (Modified Scheuer method) & Cases (\%) \\
\hline 1 & $\begin{array}{l}(0+1+0) \\
(1+0+0)\end{array}$ & 18 \\
\hline 2 & $(1+1+0)$ & 12 \\
\hline 3 & $(1+1+1)$ & 04 \\
\hline 4 & $(2+1+1)$ & 01 \\
\hline 5 & $(3+1+1)$ & 02 \\
\hline 6 & $(2+3+1)$ & 04 \\
\hline 7 & $(2+3+2)$ & 03 \\
\hline
\end{tabular}

Column 1 indicates portal/periportal inflammation

Column 2 indicates lobule inflammation

Column 3 indicates degree of fibrosis

\begin{tabular}{|l|l|l|}
\hline 1 & Grade & \\
\hline & A & Portal inflammation and interface hepatitis \\
\hline & 0 & Absent or minimal \\
\hline & 1 & Portal inflammation only \\
\hline & 3 & Mild or localized interface hepatitis \\
\hline & 4 & Moderate or more extensive interface hepatitis \\
\hline & B & Severe and widespread interface hepatitis \\
\hline & 0 & Lobular activity \\
\hline & 1 & None \\
\hline & 2 & Inflammatory cells but no hepatocellular damage \\
\hline & 3 & Focal necrosis or apoptosis \\
\hline & 4 & Severe hepatocellular damage \\
\hline
\end{tabular}




\begin{tabular}{|l|l|l|}
\hline $\mathbf{1}$ & Grade & \\
\hline $\mathbf{2}$ & Stage & \\
\hline & 0 & No fibrosis \\
\hline & 1 & Fibrosis confined to portal tracts \\
\hline & 2 & Periportal or portal-portal septa but intact vascular relationships \\
\hline & 3 & Fibrosis with distorted structure but no obvious cirrhosis \\
\hline & 4 & Probable or definite cirrhosis \\
\hline
\end{tabular}

Abbreviations:

$M=$ Male

$F=$ Female

$N A S H=$ Non Alcoholic Steatohepatitis

$D A L Y=$ disability adjusted life years

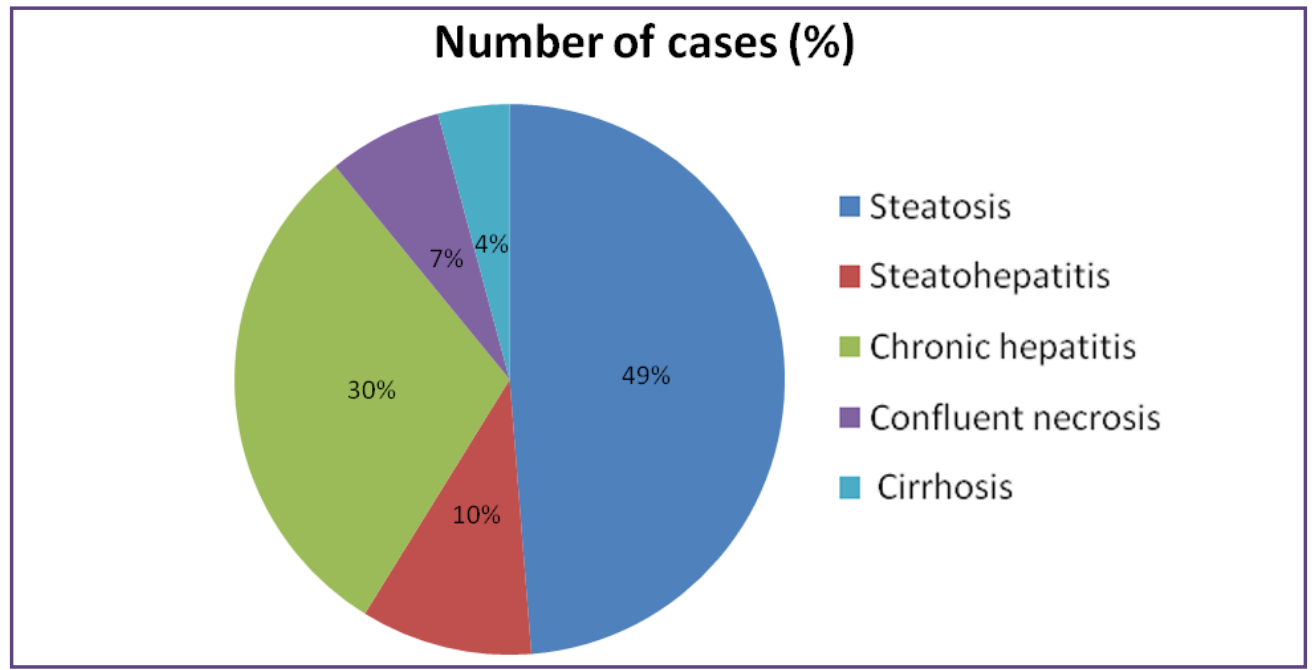

Fig.1: Distribution of various liver diseases.

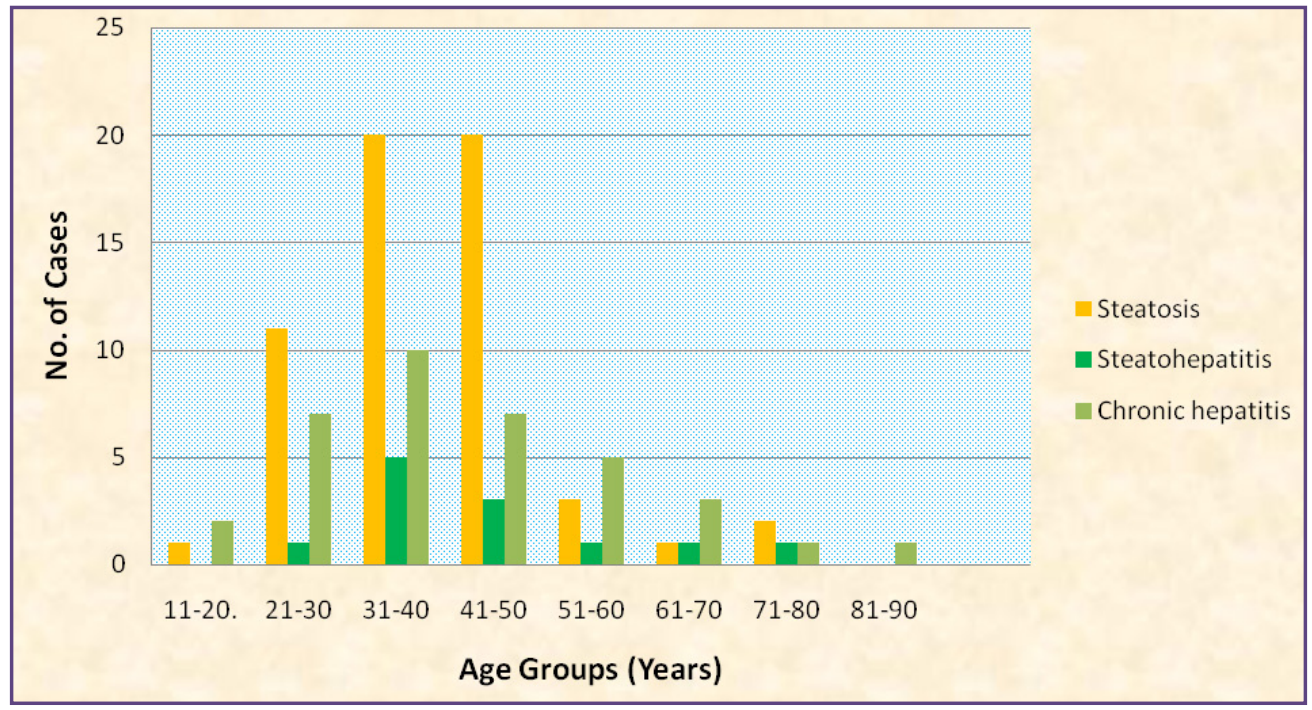

Fig. 2: Age wise distribution of Steatosis, Steatohepatitis and Chronic hepatitis.

Annals of Pathology and Laboratory Medicine, Vol. 7, Issue 5, May, 2020 


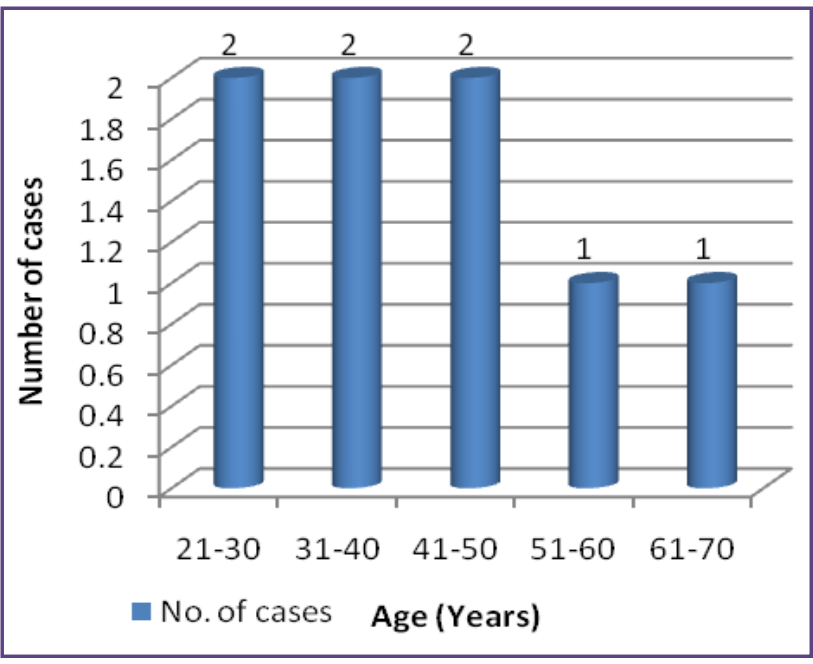

Fig. 3: Agewise distribution of Confluent necrosis.

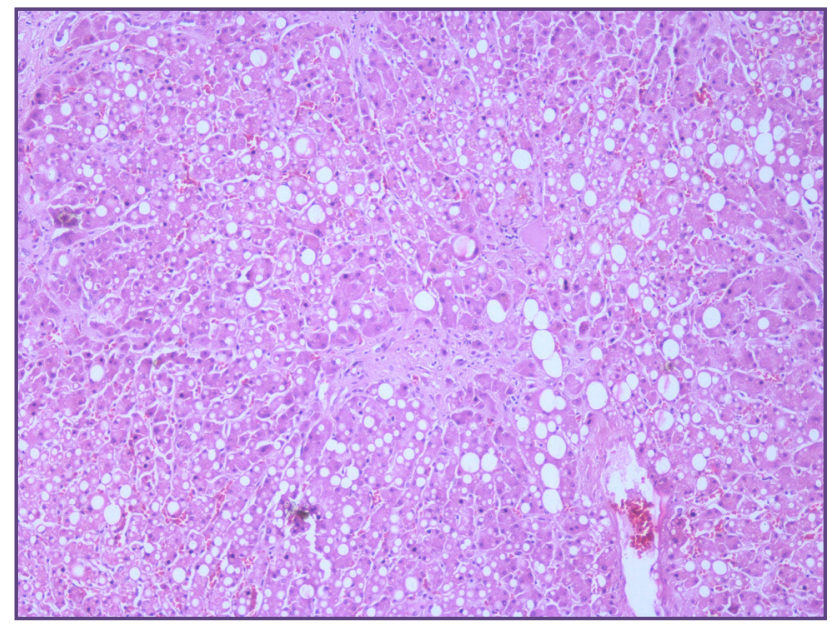

Fig. 5: H \& E stained liver section 10x view - Cirrhosis.

\section{Discussion}

The importance of silent liver disease in the overall perspective of pathology and clinical medicine cannot be overemphasized. Histopathology is the most important and useful way of diagnosing liver diseases as some may remain silent and diagnosed only at autopsy. ${ }^{[1]}$ In this prospective study we found that liver diseases are one of the common diseases in the population of Ajmer, Rajasthan.

Observed age \& sex wise distribution of cases showed that the incidences were higher in 4 th to 6 th decades of life. In studies conducted by Bal MS et $\mathrm{a}^{\left[{ }^{12]}\right.}$ and Fubara $\mathrm{S}$ et $\mathrm{al}^{[13]}$, it was observed that the commonest affected age group was $41-50$ years $(53.85 \%)$ and $41-49$ years $(28 \%)$ respectively which is comparable to the findings of the present study. The particular characteristics of forensic autopsies are the relatively young age of subjects and usually better general health condition before death. Among our cases male

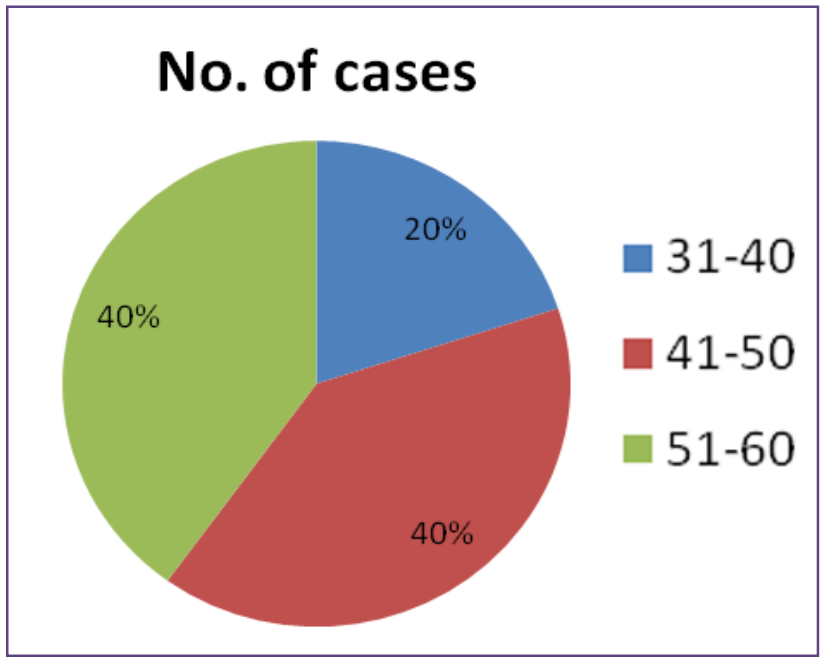

Fig. 4: Agewise distribution of Liver cirrhosis.

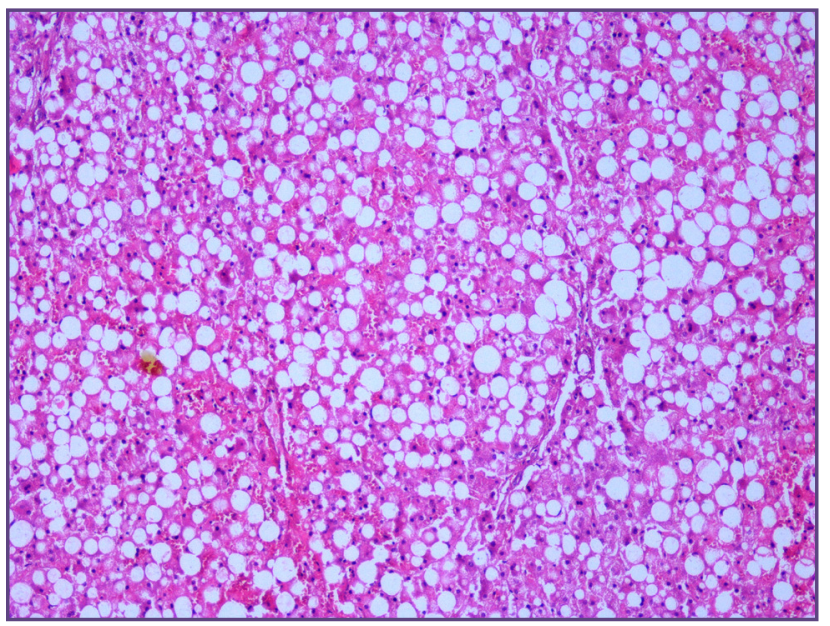

Fig. 6: H \& E stained liver section 10x view - Steatohepatitis.

gender was predominant (84.87\%), which is comparable to study done by Akhilesh Pathak \& Mangal H.M. ${ }^{[14]}$ This may be attributed to the fact that men indulge themselves more to alcohol and smoking as compared to women. Most of the subjects had no history of chronic diseases, and in more than $70 \%$ of the cases the cause of death was acute events like road traffic accident, trauma and poisoning.

Regular intake of alcohol between 40-80 grams increases the liver weight, frequency of fatty liver. These diseases are presently the most common chronic liver disease problem in western and developing countries like India. Short term ingestion of up to $8 \mathrm{gm}$ of alcohol generally produce mild to severe hepatic changes such as fatty liver, ingestion increase up to $160 \mathrm{gm}$ or more per day for more than 20 years results in cirrhosis of liver. ${ }^{[15]}$

The most common cause of acquired metabolic disorder is Non-alcoholic fatty liver disease (NAFLD) in individuals 
who do not consume alcohol or do so in a very small quantity. It includes a spectrum of liver diseases, ranging from simple steatosis to steatohepatitis, advanced fibrosis, and cirrhosis. Non-alcoholic steatohepatitis (NASH) represents a stage in the spectrum of NAFLD, characterized by presence of inflammation leading to gradual fibrosis in some of the affected patients. This disease is presently the most common chronic liver problem in western societies. According to a recent study, the prevalence of NAFLD and NASH in western general population is approximately $20 \%$ and $30 \%$ respectively. But the true incidence and prevalence of NAFLD and NASH are not well known in different populations. ${ }^{[16]}$ This is partly because liver histology is required as the gold standard for precise diagnosis of this condition and the relatively invasive procedure of liver biopsy is still not considered essential for management of NAFLD by many physicians. ${ }^{[17]}$

Most of the reports about the prevalence rates of this disease are based on ultrasonographic studies and/or the presence of elevated serum levels of aminotransferases in the absence of any other known liver disease or significant alcohol consumption. ${ }^{[18]}$

In clinical practice, diagnostic liver biopsy is only performed for highly selected patients. Therefore, the reported rates which are based on liver biopsies cannot reflect the true prevalence of NAFLD in the general population. Autopsies, performed for those who have passed away for reasons other than liver diseases, are certainly better sources for determination of a more reliable prevalence of NAFLD and NASH. In our study we found only seven known cases of alcoholics. In the rest of cases the hepatic changes were associated with NAFLD.

As regards the pattern of liver diseases, many workers ${ }^{[12,18-}$ ${ }^{23]}$ have observed that hepatic steatosis was the commonest finding. We also had steatosis as the commonest entity (48.74\%) followed by chronic hepatitis (30.25\%). Steatosis is abnormal accumulation of triglycerides in parenchymal cells, often seen in hepatocytes. Steatosis generally seen in obesity, protein malnutrition, hypoxia, diabetes mellitus and certain toxin toxicity. It occurs in these cases either due to insulin resistance or impaired GTT or impaired fasting glucose. Kringsholm B et al ${ }^{[22]}$ and Passarino $G$ et al ${ }^{[23]}$ found chronic hepatitis to be the second most common liver disease in their study. Eight cases of confluent necrosis were also observed. Cirrhosis was the least common to be observed (4.20\%). This incidence is more compared to previous studies in the literature. ${ }^{[18,24]}$ Steatosis, steatohepatitis and chronic hepatitis and confluent necrosis were common in adult between 31-40 years of age while cirrhosis was common in eld.erly person 41-60 years of age.
In a study conducted by Tsokas $\mathrm{M}$ and Tusk EE, ${ }^{[25]}$ where 45 cases of sudden death were autopsied, cirrhosis was observed in all cases $(100 \%)$ with a male: female ratio of 1.6:1. Hence, cirrhosis was found to be much higher compared to the present study and the probable cause of cirrhosis was alcohol as the toxicological analysis in these patients showed high alcohol content in the venous blood. Liver cirrhosis was coincidentally diagnosed in $13.5-40 \%$ of patients at autopsy by Iwamura $\mathrm{K}$ and Inaba R. ${ }^{[26]}$ This could be due to the fact that the morphologic changes in the liver do not occur suddenly in a short span of time and that the morphogenesis goes on insidiously. Voinova $\mathrm{LV}^{[3]}$ observed that steatosis was the most common alcohol related damage in the liver and cirrhosis in case of viral diseases. Hence detailed examination and periodic followups are necessary for early diagnosis of cirrhosis.

Cirrhosis is the common liver diseases in India. Silent liver diseases are very common amongst the apparently healthy individuals and if not detected early; some of these conditions may lead to serious outcomes. Hence, steps should be taken up for the early detection and treatment of such ailments. The study was conducted only on specimens collected from the mortuary and may not reflect the actual pattern of liver diseases and emphasizes the need for further studies for early detection and treatment of the vulnerable group of people in the local place.

\section{Conclusion}

It may be concluded from the present study that cirrhosis, chronic hepatitis and steatosis are the common liver diseases in India. Silent liver diseases are very common amongst the apparently healthy individuals and if not detected early some of these conditions may lead to serious outcomes. Hence, steps should be taken up for the early detection and treatment of such ailments. The study was conducted only on specimens collected from the mortuary and may not reflect the actual pattern of liver diseases and emphasizes the need for further studies for early detection and treatment of the vulnerable group of people in the local population.

\section{Acknowledgements}

Dr. Neena Kasliwal, Senior Professor, Department of Pathology

Mr. Anil Joshi Technician, Department of Pathology

Mr. Govind Ji, Technician, Department of Pathology

Mr. Ravikant Ji, Technician, Department of Pathology

Funding

None 


\section{Competing Interests}

None declared

\section{Reference}

1. Sotoudehmanesh R, Sotoudeh M, Ali-Asgari A, AbediArdakani B, Seyed-Mohammad T, Ali Khakinejad, et al. Silent Liver Diseases in Autopsies from Forensic Medicine of Tehran. Arch Iranian Med. 2006;9(4):324-8

2. Carithers RL Jr. Alcoholic hepatitis and cirrhosis. In Kaplowitz N(ed): Liver and Biliary diseases. Baltimore, Williams \& Wilkins, 1992, pp. 334-46.

3. Voinova LV. Aetiological and nosological structure of liver diseases (on autopsy data of clinics of I.M.Sechenov Moscow Medical Academy in 1988-1997). Arkh patol. 2000; 62: 45-47.

4. John T Galambos: liver in 4th Ed., Gastroenterology, WB Saunders Company, Pliladelphia, 1985; 2985-3048.

5. Amarapurkar DN, Hashimoto E, Lesmana LA, Sollano JD, Chen PJ, Goh KL; Asia-Pacific Working Party on NAFLD. How common is non-alcoholic fatty liver disease in the Asia-Pacific region and are there local differences? J Gastroenterol Hepatol 2007; 22: 788-793.

6. Vernon G, Baranova A, Younossi ZM. Systematic review: the epidemiology and natural history of non-alcoholic fatty liver disease and non-alcoholic steatohepatitis in adults. Aliment Pharmacol Ther 2011;34: 274-285.

7. Melato M, Sasso F, Zanconati F. Liver cirrhosis and liver cancer. A study of their relationship in 2563 autopsies. Zentralbl Pathol 1993; 139: 25-30.

8. Graudal N, Leth P, Marbjerg L, Galloe AM. Characteristics of cirrhosis undiagnosed during life: a comparative analysis of 73 undiagnosed cases and 149 diagnosed cases of cirrhosis, detected in 4929 consecutive autopsies. J Intern Med 1991; 230:165-171.

9. Lim YS, Kim WR. The global impact of hepatic fibrosis and end-stage liver disease. Clin Liver Dis 2008; 12: 733-746.

10. Mathers C, Lopez A, Murray C. The burden of disease and mortality by condition: data, methods, and results for 2001 . In: Lopez A, Mathers C, Ezzati M, et al, editors. Global burden of disease and risk factors. Washington (DC): Oxford University Press and the World Bank; 2006. p. 45-93.

11. Umesh Babu R, Gayathri B.N, Harendra Kumar M.L Spectrum of liver pathology at autopsy. International Journal of Research and Review 2015;2(3):79-86.

12. Bal MS, Singh SP, Bodal VK, Oberoi SS, Surinder K.
Pathological findings in liver autopsy. Journal of Indian Academy of Forensic Medicine 2004; 26(2):971-73

13. Fubara DS, Jebbin NJ. Hepatocellular carcinoma in Port Harcourt, Nigeria: Clinicopathologic Study of 75 Cases. Annals of African Medicine 2007; 6(2):54- 7

14. Akhilesh Pathak, Mangal H.M. Original research paper Histo-Pathology Examination in Medico-legal Autopsy Pros \& Cons. J Indian Acad Forensic Med, 2010; 32(2):128.

15. Saphir O. Liver 4th ed. Autopsy diagnosis and techniques by Paul B, Hobber, New York. 1958:354-65.

16. Copeland AR. Sudden death in the alcoholics. Jr. Forens. Sci. Inter. 1985;29:159-159

17. Hultcrantz R, Glaumann H, Lindberg G, Nilsson LH. Liver investigation in 149 asymptomatic patients with moderately elevated activities of serum aminotransferases.

18. Selvi RT, Selvam V, Subramanium PM. Common Silent liver Diseases In and Around of Salem Population: An Autopsy study. Journal of Clinical and Diagnostic Research 2010 Apr; 6(2):207-10.

19. Merat S, Sotoudehmanesh R, Nouraie M, Peikan-Heirati M, Sepanlou SG, Malekzadeh R et al. Sampling Error in Histopathology Findings of Nonalcoholic Fatty Liver Diseases: A Postmortem Liver Histology Study. Archives of Iranian Medicine 2012 July; 7:418-20.

20. Kringsholm B, Christoffersen P. Liver pathology in fatal drug addiction. Forensic Science International 1982 SepOct; 20(2): 14151.

21. Elayassi H. Fatty Liver, a postmortem study. Medical Journal of Iran Hospital 2000 Jul; 5(1):28-29.

22. Kringsholm B, Christoffersen P. Liver pathology in fatal drug addiction. Forensic Science International 1982 SepOct; 20(2): 14151.

23. Passarino G, Ciccone G, Siragusa R, Tappero P, Mollo F. Histopathological Findings in 851 Autopsies, with Toxicological and Virological Correlations. American Journal of Forensic Medicine and Pathology 2005 June; 26(2):106-16.

24. Kochar N, Lowes J, Teague RH. Nonalcoholic fatty liver disease in South West England. Gasteroenterology. 2002; 122:667-670.

25. Tsokos M, Turk EE. Esophageal variceal haemorrhage presenting as sudden death in outpatients. Archives Pathol Lab Med 2000 Oct; 126:1197-00.

26. Iwamura K, Inaba R. Clinical study on latent cirrhosis of the liver. Tokai Journal Exp Clin Med 1983 Jul; 8(3):281-91.

*Corresponding author:

Dr. Vandana Porwal, Postal Address: 450/30, opposite old temple, Mayo link road. Ajmer (Rajasthan) - 305001 India

Phone: +91 9460355266

Email: vandana2067@gmail.com

Financial or other Competing Interests: None. 